\title{
Infection of myocytes with chlamydiae
}

\author{
Guqi Wang, ${ }^{1}$ Frank Burczynski, ${ }^{1}$ Brian Hasinoff' and Guangming Zhong ${ }^{2}$
}

Author for correspondence: Guangming Zhong. Tel: +1 210567 1169. Fax: +1 2105670293.

e-mail: Zhongg@UTHSCSA.edu

1 Faculty of Pharmacy, University of Manitoba, Winnipeg, Manitoba R3T 2N2, Canada

2 Department of Microbiology and Immunology, University of Texas Health Science Center at San Antonio, 7703 Floyd Curl Drive, San Antonio, TX 78229, USA

\begin{abstract}
Chlamydial infection has been associated with myocarditis in animals and humans. However, the mechanism resulting in myocarditis following infection is not known. Here, evidence is presented that both Chlamydia trachomatis and Chlamydia pneumoniae can infect and replicate in myocytes isolated from neonate rats. The infected myocytes contained chlamydial inclusions, indicative of chlamydial growth, and infectious particles were recovered from the infected myocytes. It was also found that chlamydial infection at a late stage induced significant damage to the infected myocytes, as evidenced by an increased lactate dehydrogenase release, reactive oxygen species production and a reduced ATP level. However, no nuclear apoptosis was detected in the infected myocytes. Collectively, these observations have demonstrated that Chlamydia spp. are able to both infect and damage myocytes, suggesting a potential role of chlamydial infection in myocarditis.
\end{abstract}

Keywords: myocarditis, myocytes, chlamydial infection, apoptosis

\section{INTRODUCTION}

Chlamydiae are obligate intracellular pathogens that have to replicate inside a cytoplasmic vacuole of eukaryotic cells. There are three major species of Chlamydia. Chlamydia psittaci is a respiratory pathogen that primarily infects animals, while Chlamydia trachomatis and Chlamydia pneumoniae are human pathogens. C. trachomatis mainly causes ocular and urogenital infections, while C. pneumoniae infects the respiratory tract. More importantly, C. pneumoniae infection has been associated with vascular atherosclerosis (Blessing et al., 2001; Fong et al., 1999; Grayston, 2000; Kalayoglu et al., 2000; Laurila et al., 1997). We have previously demonstrated that respiratory infection with C. pneumoniae but not $C$. trachomatis can significantly exacerbate atherosclerotic lesions in mouse aorta (Hu et al., 1999; Liu et al., 2000).

Despite the apparent differences in tissue tropism between the chlamydial species, all three major species have been shown to be associated with or to induce myocarditis (Bachmaier et al., 1999; Blessing et al., 2000; Diaz \& Collazos, 1997; Fan et al., 1999; Gnarpe et al., 1997; Schinkel et al., 2000). Infection with C. psittaci has been demonstrated to not only cause endocarditis in

Abbreviations: i.f.u., inclusion-forming unit(s); LDH, lactate dehydrogenase. animals but is also infrequently associated with myocarditis in humans working with infected animals (Schinkel et al., 2000). Epidemiological investigations have presented evidence for a role of C. pneumoniae infection in myocarditis and sudden unexpected cardiac death. Not only are C. pneumoniae-specific antibody levels increased in patients with inflammatory heart muscle diseases (Gnarpe et al., 1997), but C. pneumoniae antigens have also been detected in endomyocardial biopsy samples (Wesslen et al., 1992). A C. trachomatis murine strain was demonstrated to cause myocarditis in mice (Bachmaier et al., 1999; Fan et al., 1999). An autoimmune hypothesis was proposed as the potential mechanism of the Chlamydia-induced myocarditis (Bachmaier et al., 1999; Diaz \& Collazos, 1997). However, chlamydial organisms were detected in the cardiac tissues of infected subjects, suggesting that the organisms may be able to invade the heart tissues. Thus, it is important to determine whether Chlamydia can actually infect myocytes and cause direct damage to the cardiac tissues. In the present study, we have used rat neonatal myocytes as the model cells to address these questions. We found that C. trachomatis (LGV) and C. pneumoniae (AR39) successfully infected myocytes and produced infectious progeny in myocytes. The chlamydial infection induced lactate dehydrogenase (LDH) release, a clinical indicator of myocarditis, and superoxide production, a known agent that can damage cardiac tissues. The Chlamydia-induced myocyte damage is accompanied with a reduced ATP level. However, 
no apparent nuclear apoptosis was detected in the infected myocytes, despite the obvious cellular damage.

\section{METHODS}

Preparation of neonate cardiomyocytes. A myocyte isolation procedure as described previously (Elmadhoun et al., 2001) was followed in this study. Briefly, hearts from 1- to 2-day-old neonatal Sprague-Dawley rats were sterilely harvested and minced into $\sim 1 \mathrm{~mm}^{3}$ pieces on ice, followed by an initial digestion with $0.1 \%$ collagenase and $0.15 \%$ trypsin (Worthington) in $\mathrm{Ca}^{2+}, \mathrm{Mg}^{2+}$-free PBS containing $1 \%$ glucose at $37^{\circ} \mathrm{C}$ for $10 \mathrm{~min}$. After discarding the initial cell-containing supernatant fluid, six additional $10 \mathrm{~min}$ digestions were carried out with the same amount of enzymes in DMEM/F-12 medium (abbreviated as DF-12; Life Technologies) with $20 \%$ fetal bovine serum (FBS). The cell-containing supernatant fluids from all six digestions were collected and the cells were washed twice with DF-12 by spinning at $300 \mathrm{~g}$ for $5 \mathrm{~min}$. Nonmyocyte cells were removed by a discontinuous Percoll gradient centrifugation. The cell viability was assessed by trypan blue exclusion and was typically greater than $95 \%$. The isolated cardiomyocytes were cultured in DF-12 with $10 \%$ FBS at a density of $0 \cdot 2-0 \cdot 5 \times 10^{6}$ cells per well of a $24-w e l l$ plate in an incubator supplied with $5 \% \mathrm{CO}_{2}$. The culture medium was replenished daily. A unique characteristic of the myocytes is that an obvious cellular beating can be seen under an inverted microscope. We found that all visible cell mass uniformly showed beating prior to infection. Four days after culturing, the myocytes were subjected to infection with chlamydial organisms.

Chlamydial organisms and infection. The C. trachomatis LGV (serovar L2) and C. pneumoniae AR39 strains were obtained from Washington Research Foundation, Seattle, USA, and were propagated and purified as described previously (Hu et al., 1999; Zhong et al., 2001). Aliquots of the organisms were frozen at $-80^{\circ} \mathrm{C}$ before being used for infection. For AR39 infection, the organisms were diluted in DMEM without FBS and inoculated onto cell monolayers in a volume of $200 \mu \mathrm{l}$ per well at an m.o.i. of 2 , or as indicated in individual experiments. After incubation for $2 \mathrm{~h}$ at $37^{\circ} \mathrm{C}$, the inoculating solution was removed and $1 \mathrm{ml}$ of DMEM with $10 \%$ FBS was added to each well. For L2 infections, the organisms were directly diluted in 10\% FBS/DMEM, and $1 \mathrm{ml}$ of the organism-containing solution was added to each well. The organisms were allowed to grow for various periods of time, as indicated in individual experiments. No cycloheximide was added to any of the myocyte infection cultures. However, $2 \mu \mathrm{g}$ cycloheximide $\mathrm{ml}^{-1}$ was kept in the culture medium used for titrating the chlamydial organisms in HeLa cells. For determining the burst size of the myocyte-born chlamydial inclusions, lysates harvested from the infected myocytes were serially diluted in PBS and inoculated onto HeLa cell monolayers grown on coverslips. Forty-eight (for L2-infected myocyte lysate sample) or 72 (for AR39-infected myocyte lysate sample) hours post-infection, the coverslips were fixed for immunofluorescence staining as described below and the number of chlamydial inclusions (also designated as inclusion-forming units, i.f.u.) per view was counted for 10 random views from duplicate coverslips. The total number of i.f.u. generated from a given myocyte lysate sample was calculated based on the lysate volume, dilution factors and number of views per coverslip. Finally, the burst size of the inclusions grown in myocytes was determined by dividing the total number of i.f.u. obtained from the titration of the myocyte lysates on HeLa cells by the number of i.f.u. used to initially infect myocytes.

Immunofluorescence staining. The myocyte samples (with or without infection) grown on coverslips in 24-well plates were fixed with a $2 \%$ paraformaldehyde/PBS solution for $30 \mathrm{~min}$ at room temperature, followed by permeabilization with $0.5 \%$ saponin for $30 \mathrm{~min}$ at room temperature. After blocking with $1 \%$ milk/PBS for $1 \mathrm{~h}$ at room temperature, a mouse antichlamydial LPS mAb (G. Zhong et al., unpublished data) was used to stain chlamydial antigens. The first antibody staining was visualized with a goat-anti-mouse IgG conjugated with Cy3 (red; Jackson Immunologicals). A DNA dye, Hoechst (blue; Sigma), was used to simultaneously reveal host-cell nuclei. Images were acquired individually for each staining using a SesiCam Imaging System (The Cooke Corporation, NY, USA) connected to a Nikon diaphot inverted microscope and the single colour images were merged in-frame into the dual colour image using the software SIMPLEPCI. However, for publication purposes, all colour images were flattened into black and white pictures. As a result, only the Cy3 staining (red) of chlamydial inclusions was shown as white on a black background.

LDH release assay. LDH activity was measured spectrophotometrically as described by Bergmeyer \& Bernt (1974). Aliquots of the culture supernatants from each well were collected and centrifuged for $5 \mathrm{~min}$ at $500 \mathrm{~g}$. The supernatant was saved in a sterile microcentrifuge tube and stored at $-80{ }^{\circ} \mathrm{C}$. All solutions used for this assay were made fresh on the day of the assay. The substrate for the $\mathrm{LDH}$ reaction was made by mixing $10 \%(\mathrm{v} / \mathrm{v}) \mathrm{NADH}$ and $10 \%(\mathrm{v} / \mathrm{v})$ pyruvate in Tris/ $\mathrm{KCl}$ buffer and equilibrated to $25^{\circ} \mathrm{C}$ in a water bath prior to use. Quadruplicate $20 \mu \mathrm{l}$ aliquots of the supernatants (containing LDH) were added to quartz cuvettes containing $800 \mu \mathrm{l}$ of the substrate solution in a Cary Win UV spectrometer. The change in absorbance at $340 \mathrm{~nm}$ is directly proportional to LDH activity in the supernatant samples.

Detection of ATP levels. Rat cardiomyocytes with or without chlamydial infection were rinsed twice with cold PBS, and then treated with lysis buffer $(0 \cdot 1 \mathrm{M}$ potassium phosphate, $1 \%$ Triton X-100, $1 \mathrm{mM}$ DTT, $2 \mathrm{mM}$ EDTA, pH 7.8) for $15 \mathrm{~min}$ at $65^{\circ} \mathrm{C}$. The cell lysates were collected and frozen at $-80^{\circ} \mathrm{C}$. The ATP levels in the lysates were determined using a standard firefly luciferase-luciferrin bioluminescent assay Kit (Sigma) on a Beckman LS 6500 multi-purpose scintillation counter with single photo monitor. The results were expressed as ATP concentration $(\mathrm{nM})$. Each measurement was carried out in duplicate and two experiments were performed.

Assessment of oxidative stress. The intracellular oxidative stress in cardiomyocytes was assessed by monitoring the oxidation of 2,7-dichlorofluorescein diacetate (H2DCF) to highly fluorescent dichlorofluorescein (Swift \& Sarvazyan, 2000). Briefly, a $10 \mathrm{mM}$ stock solution of H2DCF (Sigma) was freshly dissolved in ethanol and kept at $-20^{\circ} \mathrm{C}$. The stock solution was diluted to $10 \mu \mathrm{M}$ with PBS (containing both $\mathrm{Ca}^{2+}$ and $\mathrm{Mg}^{2+}$ ) prior to the experiment. Rat neonate myocytes were washed twice with warm $\mathrm{Ca}^{2+}, \mathrm{Mg}^{2+}$-containing PBS and incubated with $10 \mu \mathrm{M} \mathrm{H} 2 \mathrm{DCF}(0.5 \mathrm{ml}$ per well of a $24-w e l l$ plate) at room temperature in the dark. After $20 \mathrm{~min}$ incubation, cells were washed twice with PBS to remove the extracellular probe, and image analysis (488 $\mathrm{nm}$ excitation wavelength, $515 \mathrm{~nm}$ emission wavelength) was conducted immediately using an inverted Nikon microscope with an Axon Integrated Imaging System (Axon Instruments). All images were collected at room temperature. Mean fluorescence intensity of individual cells was calculated by Axon Imaging Workbench Software and the images were 
presented in grey scale so that the degree of the intracellular fluorescence brightness is directly proportional to the level of oxidative species in individual cells.

\section{RESULTS}

\section{C. trachomatis (L2) and C. pneumoniae (AR39) can infect rat myocytes}

Myocyte samples were infected with C. trachomatis L2 or C. pneumoniae AR39, and infection was detected with an immunofluorescence staining assay. As shown in Fig. 1, inclusions filled with chlamydial antigens were detected in the cytosol of myocytes infected with either L2 (at 24 or 48 h post-infection) or AR39 (at 48 h or $72 \mathrm{~h}$ post-infection). Since chlamydial infection starts with the entry of a single elementary body $(\sim 0.2 \mu \mathrm{m})$ into a host cell via endocytosis, a sizeable cytoplasmic inclusion is an indication of chlamydial organism replication inside myocytes. Prior to fixation, we observed that myocytes harbouring chlamydial inclusions were still able to beat, a characteristic of viable cardiomyocytes, although at a lower rate compared to the uninfected cells (data not shown). To determine whether the infected myocytes produce infectious progeny, myocytes infected with either L2 (48 h post-infection) or AR39 (72 h post-infection) were harvested for two additional measurements. First, the infected cell pellets were used for making ultrathin sections for electron microscopic observation. Clear infectious forms of chlamydial organisms were seen in both L2- and AR39infected myocytes (data not shown). However, the static
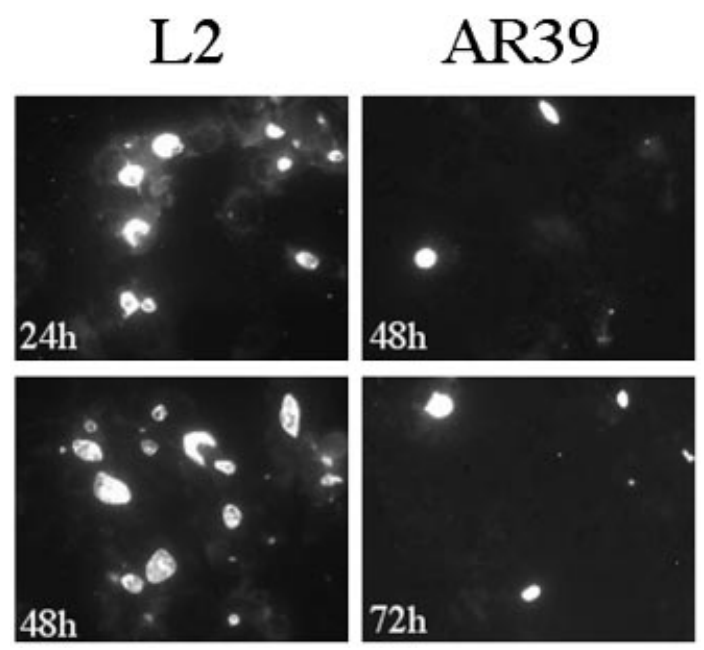

Fig. 1. Infection of myocytes with L2 and AR39. Myocytes were infected and at various time points post-infection, as indicated in each panel, the infected cells were stained with a Chlamydiaspecific antibody (bright; visualized with a Cy3-conjugated secondary antibody) and a DNA dye for visualizing host-cell nuclei (not shown due to black and white presentation). Chlamydial inclusions were detected in rat neonate myocytes infected with either $C$. trachomatis (left panels) or $C$. pneumoniae (right panels).

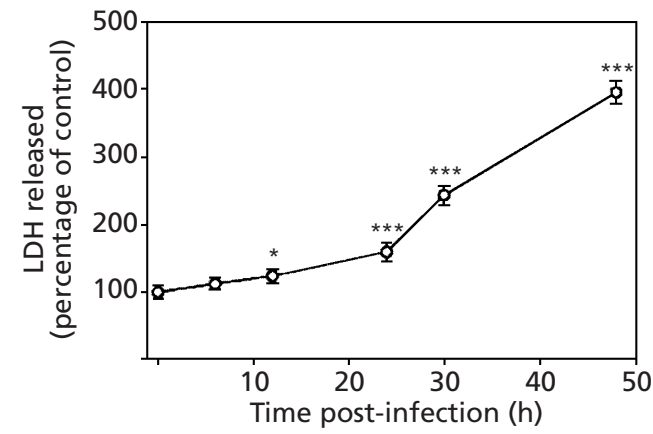

Fig. 2. LDH release from Chlamydia-infected myocytes. Myocytes were infected with C. trachomatis L2 at an m.o.i. of 2, and the culture supernatants were harvested at various times post-infection. The results were measured as the absorbance at $340 \mathrm{~nm}$, expressed as the rate of absorbance reduction, and plotted as the percentile of the absorbance reduction rate from the corresponding control groups. Each set of measurements was carried out in quadruplicate and four experiments were performed. Asterisks denote statistically significant differences between the infected and uninfected control samples at the corresponding time points $(*, P<0.05 ; * * *, P<0.001$; Student's t-test with two tails).

microscopic observation alone could not allow us to determine whether the infectious particles were still alive. We sonicated the parallel cell pellet samples for measuring the infectivity of the released intracellular particles. The sonicated myocyte lysates were serially diluted in PBS and used to infect HeLa cell monolayers, as described in Methods. The myocyte lysates produced obvious inclusions in HeLa cells (data not shown). We found that the lysates harvested from myocytes infected with $1 \times 10^{6}$ i.f.u. of AR39 organisms generated $\sim 2 \times 10^{7}$ i.f.u. when titrated in HeLa cells, while the lysates of myocytes similarly infected with L2 generated $\sim 1 \times 10^{8}$ i.f.u. Therefore, the burst sizes of AR39 and L2 in myocytes are 20 and 100, respectively. We can, therefore, conclude that $C$. trachomatis and $C$. pneumoniae can cause productive infection in rat neonate myocytes.

\section{Chlamydial infection damages myocytes}

We next assessed the effects of chlamydial infection on myocyte integrity. Since detection of LDH in blood samples is useful for the diagnosis of myocarditis, we used the LDH release assay to evaluate the potential damage of chlamydial infection on myocytes at various time points post-infection. As shown in Fig. 2, a significant increase in LDH release was found in myocytes $12 \mathrm{~h}$ post-infection with C. trachomatis L2 $(P<0.05$, by comparing to the uninfected control cell samples). As infection continued, more LDH release was detected. These observations suggest that chlamydial infection can cause significant damage to myocyte membrane permeability. To understand how chlamydial infection causes damage to myocytes, we compared the production of oxidative species between infected and 
(a)

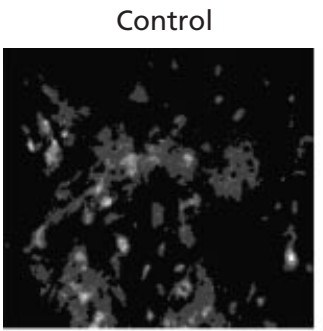

$72 \mathrm{~h}$

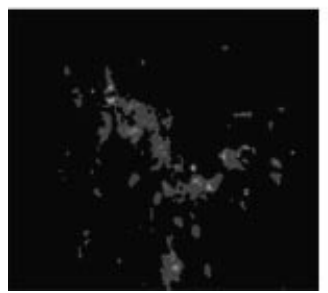

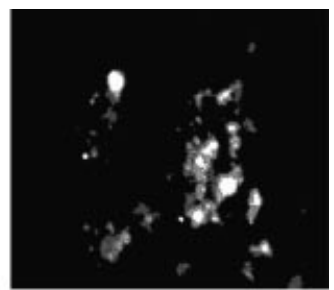

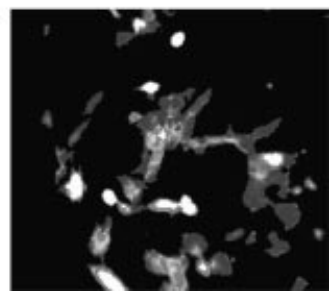

(b)

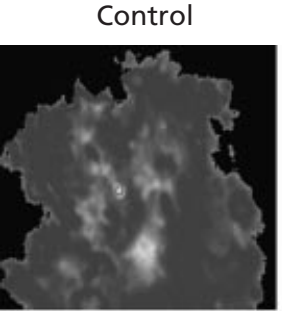

AR39 Infection
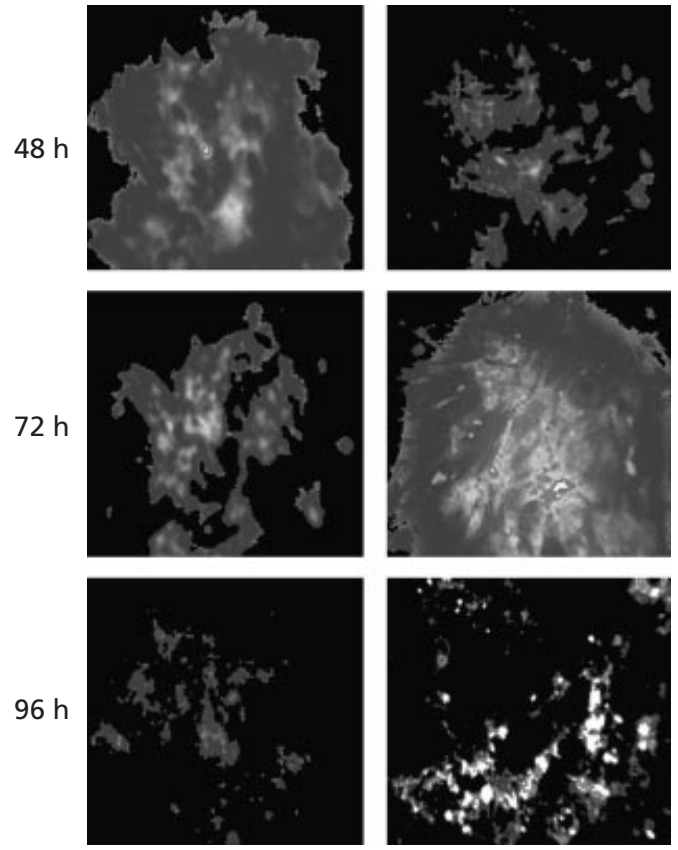

Fig. 3. Detection of oxidative species in cultures of myocytes, with or without chlamydial infection. Myocytes were infected with C. trachomatis L2 (a) or AR39 (b) at an m.o.i. of 2. At various time points post-infection, the cell samples were processed for measuring oxidative species in situ as described in Methods. The intensity of the fluorescence generated from the intracellularly oxidized dichlorofluorescein is proportional to the levels of oxidative species inside cells.

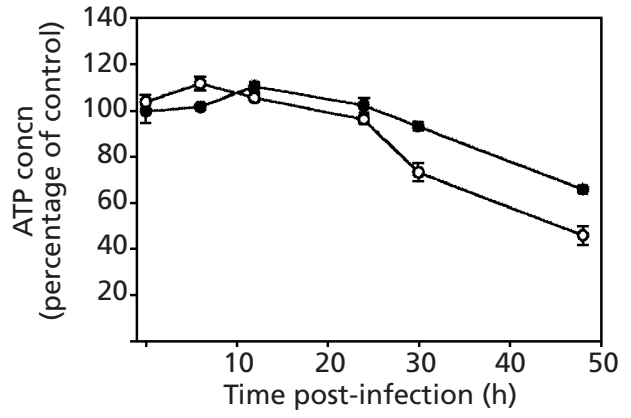

Fig. 4. Total ATP levels of myocyte samples infected with either C. trachomatis L2 (O) or C. pneumoniae AR39 (0). Myocytes were infected with Chlamydia at an m.o.i. of 2, and the samples were harvested at various time points post-infection for ATP measurements. The results were expressed as ATP concentration (nM) and plotted as a percentage of the corresponding control samples. Each measurement was carried out in duplicate and two experiments were performed.

uninfected cells (Fig. 3). The C. trachomatis L2 (Fig. 3a) and the C. pneumoniae AR39 (Fig. 3b) infections caused a progressive increase in reactive oxygen species that can oxidize the exogenous marker 2,7-dichlorofluorescein diacetate into dichlorofluorescein as a function of infection time. The fluorescence intensity of the oxidized dichlorofluorescein inside cells correlates well with the levels of intracellular oxidative species. Quantitative analysis by comparing the sizes of oxidized areas on coverslips showed that there was a $1 \cdot 5$ - to 3 -fold greater increase in oxidative species in the infected cell samples than in the uninfected samples (data not shown). Although it is not known what exactly the measured oxidative species were, it is thought that the major species are hydrogen peroxide and hydroxyl free radical, both of which are known to cause damage to myocyte membrane permeability. Since the ATP level is an indication of cellular viability, we evaluated whether chlamydial infection can affect host-cell ATP production (Fig. 4). We found that the total ATP levels were significantly lower at $30 \mathrm{~h}$ post-infection with L2 and at $48 \mathrm{~h}$ post-infection with AR39. These observations further confirmed that chlamydial infection caused significant damage to myocytes at the later stage of infection. However, despite the infection-induced damage, there was no apparent nuclear apoptosis in the infected myocytes even at a late stage of infection (Fig. 1; no apparent nuclear condensation was found), which is consistent with our previous observations that Chlamydia spp. can actively prevent host cells from undergoing apoptosis (Fan et al., 1998).

\section{DISCUSSION}

Chlamydiae are ubiquitous pathogens that can infect multiple types of hosts at various tissue sites. All three major chlamydial species have been shown to be associated with or to cause inflammation in the heart, suggesting that Chlamydia spp. may be able to infect 
heart muscle cells. Although chlamydiae are known to infect both endothelial and smooth muscle cells of the cardiovascular system (Gaydos et al., 1996), it was not known whether Chlamydia spp. could successfully infect myocytes. The present study provided the first experimental evidence showing that chlamydiae are able to productively infect myocytes and cause significant damage to the infected cells.

During epithelial infection of respiratory and urogenital tracts, the Chlamydia-induced diseases are largely due to persistent infection. It is not known whether $C$. trachomatis or C. pneumoniae can establish persistence in heart muscle cells. Nevertheless, either the direct damage caused by productive infection or the inflammatory response provoked by persistent infection in the heart can be equally fatal to the infected hosts. It is unlikely that heart muscle cells are the natural target cells for chlamydial organisms, since transmission to new hosts from this tissue is difficult. However, once chlamydial organisms are accidentally carried to the heart of the host, chlamydial growth inside the myocytes may cause damage to the heart tissue. This hypothesis is supported by our current finding that chlamydial infection in myocytes induced the production of reactive oxygen species (Fig. 3), agents known to cause cell damage, and triggered release of $\mathrm{LDH}$ (Fig. 2), an indicator of cell membrane permeability alteration.

\section{ACKNOWLEDGEMENTS}

This work was supported in part by grants from the Medical Research Council of Canada (F.B.) and the US National Institutes of Health (G.Z.).

\section{REFERENCES}

Bachmaier, K., Neu, N., de la Maza, L. M., Pal, S., Hessel, A. \& Penninger, J. M. (1999). Chlamydia infections and heart disease linked through antigenic mimicry. Science 283, 1335-1339.

Bergmeyer, H. U. \& Bernt, E. (1974). Lactate dehydrogenase UVassay with pyruvate and NADH. In Methods of Enzymatic Analysis, vol. 2, 2nd edn, pp. 574-578. Edited by H. U. Bergmeyer. New York, NY: Academic Press.

Blessing, E., Lin, T. M., Campbell, L. A., Rosenfeld, M. E., Lloyd, D. \& Kuo, C. (2000). Chlamydia pneumoniae induces inflammatory changes in the heart and aorta of normocholesterolemic C57BL/6J mice. Infect Immun 68, 4765-4768.

Blessing, E., Campbell, L. A., Rosenfeld, M. E., Chough, N. \& Kuo, C. C. (2001). Chlamydia pneumoniae infection accelerates hyperlipidemia induced atherosclerotic lesion development in C57BL/6J mice. Atherosclerosis 158, 13-17.

Diaz, F. \& Collazos, J. (1997). Myopericarditis due to Chlamydia psittaci. The role of autoimmunity. Scand J Infect Dis 29, 93-94.
Elmadhoun, B. M., Wang, G. Q., Kirshenbaum, L. A. \& Burczynski, F. J. (2001). Palmitate uptake by neonatal rat myocytes and hepatocytes. Role of extracellular protein. Eur J Biochem 268, 3145-3153.

Fan, T., Lu, H., Hu, H., Shi, L., McClarty, G. A., Nance, D. M., Greenberg, A. H. \& Zhong, G. (1998). Inhibition of apoptosis in Chlamydia-infected cells: blockade of mitochondrial cytochrome $c$ release and caspase activation. J Exp Med 187, 487-496.

Fan, Y., Wang, S. \& Yang, X. (1999). Chlamydia trachomatis (mouse pneumonitis strain) induces cardiovascular pathology following respiratory tract infection. Infect Immun 67, 6145-6151.

Fong, I. W., Chiu, B., Viira, E., Jang, D. \& Mahony, J. B. (1999). De novo induction of atherosclerosis by Chlamydia pneumoniae in a rabbit model. Infect Immun 67, 6048-6055.

Gaydos, C. A., Summersgill, J. T., Sahney, N. N., Ramirez, J. A. \& Quinn, T. C. (1996). Replication of Chlamydia pneumoniae in vitro in human macrophages, endothelial cells, and aortic artery smooth muscle cells. Infect Immun 64, 1614-1620.

Gnarpe, H., Gnarpe, J., Gastrin, B. \& Hallander, H. (1997). Chlamydia pneumoniae and myocarditis. Scand J Infect Dis Suppl 104, 50-52.

Grayston, J. T. (2000). What is needed to prove that Chlamydia pneumoniae does, or does not, play an etiologic role in atherosclerosis? J Infect Dis 181 Suppl. 3, S585-S586.

Hu, H., Pierce, G. N. \& Zhong, G. (1999). The atherogenic effects of Chlamydia are dependent on serum cholesterol and specific to Chlamydia pneumoniae. J Clin Invest 103, 747-753.

Kalayoglu, M. V., Indrawati, Morrison, R. P., Morrison, S. G., Yuan, Y. \& Byrne, G. I. (2000). Chlamydial virulence determinants in atherogenesis: the role of chlamydial lipopolysaccharide and heat shock protein 60 in macrophage-lipoprotein interactions. J Infect Dis 181 Suppl. 3, S483-S489.

Laurila, A., Bloigu, A., Nayha, S., Hassi, J., Leinonen, M. \& Saikku, P. (1997). Chronic Chlamydia pneumoniae infection is associated with a serum lipid profile known to be a risk factor for atherosclerosis. Arterioscler Thromb Vasc Biol 17, 2910-2913.

Liu, L., Hu, H., Ji, H., Murdin, A. D., Pierce, G. N. \& Zhong, G. (2000). Chlamydia pneumoniae infection significantly exacerbates aortic atherosclerosis in an LDLR $-/-$ mouse model within six months. Mol Cell Biochem 215, 123-128.

Schinkel, A. F., Bax, J. J., van der Wall, E. E. \& Jonkers, G. J. (2000). Echocardiographic follow-up of Chlamydia psittaci myocarditis. Chest 117, 1203-1205.

Swift, L. M. \& Sarvazyan, N. (2000). Localization of dichlorofluorescin in cardiac myocytes: implications for assessment of oxidative stress. Am J Physiol Heart Circ Physiol 278, H982-H990.

Wesslen, L., Pahlson, C., Friman, G., Fohlman, J., Lindquist, O. \& Johansson, C. (1992). Myocarditis caused by Chlamydia pneumoniae (TWAR) and sudden unexpected death in a Swedish elite orienteer. Lancet 340, 427-428.

Zhong, G., Fan, P., Ji, H., Dong, F. \& Huang, Y. (2001). Identification of a chlamydial protease-like activity factor responsible for the degradation of host transcription factors. J Exp Med 193, 935-942.

Received 26 July 2002; revised 20 August 2002; accepted 29 August 2002. 